\title{
Capacitively Coupled Hydrogen Discharges: Modeling vs. Experiment
}

\author{
L. Marques ${ }^{1,2}$, J. Jolly ${ }^{3}$, G. Gousset ${ }^{4}$ and L. L. Alves ${ }^{1,(a)}$ \\ ${ }^{1}$ Centro de Física dos Plasmas, Instituto Superior Técnico, 1049-001 Lisboa, Portugal \\ ${ }^{2}$ Departamento de Física, Universidade do Minho, 4710-057 Braga, Portugal \\ ${ }^{3}$ Lab. de Physique et Technologie des Plasmas, Ecole Polytechnique, 91128 Palaiseau Cedex, France \\ ${ }^{4}$ Lab. de Physique des Gaz et des Plasmas, Université Paris-Sud, 91405 Orsay Cedex, France
}

This paper presents a systematic characterization of hydrogen capacitively coupled very high frequency discharges, produced within a parallel plate cylindrical setup, by comparing numerical simulations to experimental measurements for various plasma parameters. A good quantitative agreement is found between calculation and experiment for the coupled electrical power and the plasma potential, at various frequencies, pressures and applied voltages. However, the model generally underestimates the electron density and the self-bias potential with respect to measured values. Model predictions for the absolute density of $H(n=1)$ atoms are compared to first diagnostic results, obtained by two-photon absorption laser-induced fluorescence diagnostics at various pressures and frequencies.

Keywords: Capacitively coupled discharges, radio-frequency discharges, hydrogen plasma, hydrogen atoms, kinetic model

\section{Introduction}

Capacitively coupled radio frequency (ccrf) discharges are currently used in the plasma enhanced chemical vapor deposition (pecvd) of hydrogenated microcrystalline silicon $(\mu-\mathrm{Si}: \mathrm{H})$ thin films, from a precursor mixture of $\mathrm{SiH}_{4}-\mathrm{H}_{2}$, under high dilution conditions for silane. Moreover, rf discharges working above the conventional $13.56 \mathrm{MHz}$ frequency exhibit an increasing interest in plasma assisted thin-film deposition and etching. In fact, the use of higher frequencies is normally associated to an enhancement of the plasma processes efficiency, through an increase in the population of hydrogen atoms.

The study of capacitively coupled very high frequency (ccvhf) discharges in pure hydrogen is therefore of great interest in optimizing pecvd reactors used for such applications. This

(a) Corresponding author Phone: +351-21 8419376 e-mail: llalves@alfa.ist.utl.pt 
optimization is often made empirically, following a technical procedure that turns out to be both random and lengthy. With this respect, modeling constitutes a competitive alternative answer. Systematic and fast results can be obtained by using well-validated models, to simulate the operation features of such devices over a broad range of working conditions.

This paper presents a systematic characterization of pure hydrogen ccvhf discharges, produced within a parallel plate cylindrical setup, by comparing numerical simulations to experimental measurements for various plasma parameters, at different frequencies $(f=13.56-80 \mathrm{MHz})$, pressures ( $p=0.2-1$ Torr), and $\mathrm{rf}$ applied voltages $\left(V_{\mathrm{rf}}=50-600 \mathrm{~V}\right)$.

The experimental setup used here is similar to the GEC reference cell and it has been described in detail elsewhere $[1,2]$. The rf discharge is sustained between parallel plate electrodes $(128$ $\mathrm{mm}$ diameter and $32 \mathrm{~mm}$ interelectrode distance), with the upper electrode (on which the $\mathrm{rf}$ voltage is measured) driven, and the lower electrode grounded. A grounded counterelectrode shields the back of the powered electrode, and the plasma is confined to the interelectrode volume by a cylindrical grid fixed to the counterelectrode.

The simulations use a two-dimensional (2D) fluid model, which has first adopted a simplified hydrogen kinetics to describe the dynamics of electrons and positive ions $\mathrm{H}^{+}, \mathrm{H}_{2}{ }^{+}$, and $\mathrm{H}_{3}{ }^{+}$, in the reactor under study [3,4]. This simplified model version (smv) yielded a good quantitative agreement between calculation and experiment for the coupled electrical power and the plasma potential. However, smv predictions for the plasma density and the self-bias voltage showed only a qualitatively agreement with respect to measurements. In fact, the values of these quantities, calculated with the smv, were systematically underestimated with respect to measurements $[4,5]$, which was attributed to both experimental uncertainties and the simplified hydrogen kinetics considered.

In order to clarify the role and importance of the hydrogen kinetics in the results obtained, we have self-consistently coupled the previously developed charged particle transport model with a homogeneous kinetic model for hydrogen, including vibrationally excited molecular species and electronically excited atomic species [6,7]. Calculation results obtained with this complete model version (cmv) are compared here to both experimental measurements and predictions of the smv, for the electron density $n_{e}$, the self-bias voltage $V_{\mathrm{dc}}$, the plasma potential $V_{p}$, the effective electrical power coupled to the plasma $W_{\text {eff, }}$ and the $\mathrm{H}$-atom density $n_{\mathrm{H}}$, at various pressures, excitation frequencies, and rf applied voltages. 


\section{Model formulation}

\subsection{Simplified model version}

The smv corresponds to a 2D time-dependent fluid model for electrons and $\mathrm{H}^{+}, \mathrm{H}_{2}{ }^{+}$, and $\mathrm{H}_{3}{ }^{+}$ positive ions, which solves the continuity, momentum transfer and mean energy equations (the latter for electrons only), coupled with Poisson's equation. Boundary conditions involve symmetry considerations at reactor axis, and the imposition of the different particle and energy fluxes, together with the applied rf potential, at each physical boundary (electrodes, grid). Model calculations are restricted to the volume between the electrodes, corresponding to a 2D workspace delimited by the discharge axis $(r=0)$, the grounded lateral grid $(r=R)$, the driven electrode $(z=0)$, and the grounded electrode $(z=d)$. Electron transport parameters are calculated adopting the local electron mean energy approximation [3]. This assumes that the space-time dependence of the electron energy distribution function (eedf) and its related transport parameters (obtained by solving the homogeneous and stationary electron Boltzmann equation, written in the two-term approximation) proceeds via the electron mean energy profile, as obtained from the fluid code. A detailed description of the smv and its numerical solution can be found in Refs. [3,4].

The smv adopts a simplified kinetic scheme for hydrogen, corresponding to the mechanisms marked with a cross symbol in Table I. In particular, this simplified kinetics: (i) does not explicitly include a model for $\mathrm{H}$-atoms, meaning that although the dissociation of $\mathrm{H}_{2}$ is considered as an electron energy loss channel, collisional-radiative and transport mechanisms, allowing an estimation of the $\mathrm{H}$-atoms density, are not taken into account; (ii) it neglects the production of negative ions, following the dissociative attachment of molecular hydrogen $[\mathrm{e}+$ $\left.\mathrm{H}_{2}(v=0-9) \rightarrow \mathrm{H}+\mathrm{H}^{-}\right]$.

\subsection{Complete model version}

The complete description of low-temperature non-equilibrium plasmas requires a simultaneous analysis of the charged particle transport and the gas phase chemistry. This study concerns mainly the electron population, as electron-neutral collisions are essential to establish the final chemical composition of the gas phase, by coupling the plasma to the neutral gas. Hence, the modeling of this problem should involve a microscopic analysis of the energy exchanges between interacting populations, via the calculation of their corresponding energy distribution functions. 
TABLE I. Hydrogen kinetic reactions considered in the model.

\begin{tabular}{|c|c|c|c|}
\hline & Process & smv & Refs. \\
\hline \multicolumn{4}{|l|}{ Molec. species } \\
\hline Elastic & $\mathrm{e}+\mathrm{H}_{2}(v) \rightarrow \mathrm{e}+\mathrm{H}_{2}(v)$ & $\mathrm{X}$ & {$[8,9]$} \\
\hline$e-R$ & $\mathrm{e}+\mathrm{H}_{2}(v, J) \rightarrow \mathrm{e}+\mathrm{H}_{2}(v, J+2), J=0-3$ & $\mathrm{X}$ & {$[8,9]$} \\
\hline$e-V$ & $\mathrm{e}+\mathrm{H}_{2}(v) \leftrightarrow \mathrm{e}+\mathrm{H}_{2}(v \pm i), \mathrm{i}=1-3$ & $\mathrm{X}$ & {$[8,9]$} \\
\hline$E-V$ & $\mathrm{e}+\mathrm{H}_{2}(v) \rightarrow \mathrm{e}+\mathrm{H}_{2}\left(\mathrm{~B}^{1} \sum_{\mathrm{u}}^{+}, \mathrm{C}^{1} \prod_{\mathrm{u}}\right) \rightarrow \mathrm{e}+\mathrm{H}_{2}(v)$ & $\mathrm{X}$ & {$[8,9]$} \\
\hline $\mathrm{V}-\mathrm{V}$ & $\mathrm{H}_{2}(v)+\mathrm{H}_{2}(w) \leftrightarrow \mathrm{H}_{2}(v-1)+\mathrm{H}_{2}(w+1)$ & & [9] \\
\hline $\mathrm{V}-\mathrm{T}$ & $\mathrm{H}_{2}(v)+\mathrm{H}_{2} \leftrightarrow \mathrm{H}_{2}(v \pm 1)+\mathrm{H}_{2}$ & & [9] \\
\hline $\mathrm{V}-\mathrm{T}$ & $\mathrm{H}_{2}(v)+\mathrm{H} \leftrightarrow \mathrm{H}_{2}(v \pm i)+\mathrm{H}, \quad \mathrm{i}=1 . .5$ & & {$[9,10]$} \\
\hline Triplet exc. + Diss. & $\begin{aligned} \mathrm{e}+\mathrm{H}_{2}(v) \rightarrow \mathrm{e}+ & \mathrm{H}_{2}\left(\mathrm{a}^{3} \sum_{\mathrm{g}}^{+}, \mathrm{b}^{3} \sum_{\mathrm{u}}^{+}, \mathrm{c}^{3} \prod_{\mathrm{u}}, \mathrm{e}^{3} \sum_{\mathrm{u}}^{+}\right) \\
& \rightarrow \mathrm{e}+2 \mathrm{H}(1 \mathrm{~s})\end{aligned}$ & $\mathrm{X}$ & {$[8,9]$} \\
\hline Singlet exc. + Diss. & $\begin{aligned} \mathrm{e}+\mathrm{H}_{2}(v) & \rightarrow \mathrm{e}+\mathrm{H}_{2}\left(\mathrm{D}^{1} \prod_{\mathrm{u}}, \mathrm{B}^{\prime \prime}{ }^{1} \sum_{\mathrm{u}}^{+}, \mathrm{D}^{\prime 1} \prod_{\mathrm{u}}\right) \\
& \rightarrow \mathrm{e}+\mathrm{H}(1 \mathrm{~s})+\mathrm{H}(\mathrm{n}=2,3)\end{aligned}$ & $\mathrm{X}$ & [8] \\
\hline Ionization by & $\mathrm{e}+\mathrm{H}_{2}(v) \rightarrow \mathrm{e}+\mathrm{e}+\mathrm{H}_{2}^{+}$ & $\mathrm{X}$ & {$[8-12]$} \\
\hline electron impact & $\mathrm{e}+\mathrm{H}_{2}(v) \rightarrow \mathrm{e}+\mathrm{e}+\mathrm{H}^{+}+\mathrm{H}$ & $\mathrm{X}$ & {$[8-12]$} \\
\hline Diss. attachment & $\mathrm{e}+\mathrm{H}_{2}(v) \rightarrow \mathrm{e}+\mathrm{H}_{2}^{-} \rightarrow \mathrm{H}+\mathrm{H}^{-}$ & & [13] \\
\hline Wall de-exc. & $\mathrm{H}_{2}(v>0)+$ wall $\rightarrow \mathrm{H}_{2}(v=0)$ & & [14] \\
\hline $\begin{array}{l}\text { Dissociation by } \\
\text { vibrat. pumping }\end{array}$ & $\begin{array}{c}\mathrm{H}_{2}(v=14)+\mathrm{H}_{2} \leftrightarrow 2 \mathrm{H}(1 \mathrm{~s})+\mathrm{H}_{2} \\
\mathrm{H}_{2}(v=14)+\mathrm{H} \leftrightarrow 3 \mathrm{H}(1 \mathrm{~s})\end{array}$ & & [15] \\
\hline \multicolumn{4}{|l|}{ Atomic species } \\
\hline Elastic & $\mathrm{e}+\mathrm{H}(\mathrm{n}) \rightarrow \mathrm{e}+\mathrm{H}(\mathrm{n})$ & & {$[16,17]$} \\
\hline $\begin{array}{l}\text { Elect. exc./de-exc. } \\
\text { Ionization by } \\
\text { electron impact }\end{array}$ & $\begin{aligned} \mathrm{e}+\mathrm{H}(\mathrm{n}) & \leftrightarrow \mathrm{e}+\mathrm{H}(\mathrm{m}), \quad \mathrm{n}, \mathrm{m}=1 . .5 \\
\mathrm{e} & +\mathrm{H}(\mathrm{n}) \rightarrow \mathrm{e}+\mathrm{H}^{+}\end{aligned}$ & & $\begin{array}{l}{[18]} \\
{[18]}\end{array}$ \\
\hline Radiative de-exc. & $\mathrm{H}(\mathrm{n}) \rightarrow \mathrm{H}(\mathrm{m})+h v$ & & [18] \\
\hline Deactivation & $\mathrm{H}(2 \mathrm{~s})+\mathrm{H}_{2} \rightarrow \mathrm{H}(2 \mathrm{p})+\mathrm{H}_{2}$ & & [19] \\
\hline & $\mathrm{H}(2 \mathrm{p})+\mathrm{H}_{2} \rightarrow \mathrm{H}(2 \mathrm{~s})+\mathrm{H}_{2}$ & & [18] \\
\hline Deact. + Diss. & $\mathrm{H}(2 \mathrm{~s})+\mathrm{H}_{2} \rightarrow 3 \mathrm{H}(1 \mathrm{~s})$ & & [19] \\
\hline Ass. Ionization & $\mathrm{H}(2 \mathrm{~s})+\mathrm{H}_{2} \rightarrow \mathrm{H}_{3}^{+}+\mathrm{H}+\mathrm{e}$ & & [18] \\
\hline Wall recombination & $\mathrm{H}(1 \mathrm{~s})+$ wall $\rightarrow 1 / 2 \mathrm{H}_{2}(v=0)$ & & [20] \\
\hline Wall de-excitation & $\mathrm{H}(\mathrm{n}>1)+$ wall $\rightarrow \mathrm{H}(1 \mathrm{~s})$ & & [18] \\
\hline
\end{tabular}

In the particular case of hydrogen, a realistic chemistry must include the kinetics of vibrationally excited molecules and of electronically excited atomic species. Hydrogen vibrational excited species have an important role in $\mathrm{rf}$ discharges, as a significant part of the coupled electrical power is transferred to low energy vibrational excitations. Moreover, these species are important channels for the production of both atomic hydrogen and charged particles. In view of this, and in order to describe pure hydrogen ccvhf discharges, we have developed a cmv that couples an updated smv (corresponding to a $2 \mathrm{D}$ time-dependent fluid model, describing the production, transport and destruction of electrons, positive ions $\mathrm{H}^{+}, \mathrm{H}_{2}{ }^{+}$, and $\mathrm{H}_{3}{ }^{+}$, and negative ions $\mathrm{H}^{-}$), the two-term homogeneous and stationary electron Boltzmann 
equation (written in the classical two-term approximation), and a homogenous collisionalradiative model $(\mathrm{crm})$ for the populations of $\mathrm{H}(\mathrm{n}=1-5)$ electronically excited atoms and $\mathrm{H}_{2}\left(\mathrm{X}^{1} \Sigma_{\mathrm{g}}{ }^{+}, v=0 . .14\right)$ vibrationally excited ground state molecules.

TABLE I. (cont) Hydrogen kinetic reactions considered in the model.

\begin{tabular}{|c|c|c|c|}
\hline & Process & smv & Refs. \\
\hline \multicolumn{4}{|l|}{ Charged species } \\
\hline \multirow{10}{*}{$\begin{array}{l}\text { Electron - ion } \\
\text { recombination }\end{array}$} & $\mathrm{e}+\mathrm{H}_{3}^{+} \rightarrow 3 \mathrm{H}$ & & [21] \\
\hline & $\mathrm{e}+\mathrm{H}_{3}^{+} \rightarrow \mathrm{H}_{2}+\mathrm{H}$ & $\mathrm{X}$ & {$[15,18-24]$} \\
\hline & $2 \mathrm{e}+\mathrm{H}_{3}^{+} \rightarrow \mathrm{H}_{2}+\mathrm{H}+\mathrm{e}$ & & [15] \\
\hline & $\mathrm{e}+\mathrm{H}_{2}^{+} \rightarrow \mathrm{H}(1 \mathrm{~s})+\mathrm{H}(\mathrm{n}), \mathrm{n}>1$ & $\mathrm{X}$ & [21-24] \\
\hline & $2 \mathrm{e}+\mathrm{H}_{2}^{+} \rightarrow 2 \mathrm{H}(1 \mathrm{~s})+\mathrm{e}$ & & [15] \\
\hline & $\mathrm{e}+\mathrm{H}^{+} \rightarrow \mathrm{H}(\mathrm{n})+h v$ & $\mathrm{X}$ & [21-24] \\
\hline & $2 \mathrm{e}+\mathrm{H}^{+} \rightarrow \mathrm{H}(\mathrm{n})+\mathrm{e}$ & & {$[15]$} \\
\hline & $\mathrm{e}+\mathrm{H}_{3}^{+}+$wall $\rightarrow \mathrm{H}_{2}+\mathrm{H}$ & & \\
\hline & $\mathrm{e}+\mathrm{H}_{2}^{+}+$wall $\rightarrow \mathrm{H}_{2}$ & & \\
\hline & $\mathrm{e}+\mathrm{H}^{+}+$wall $\rightarrow \mathrm{H}$ & & \\
\hline \multirow{3}{*}{$\begin{array}{c}\text { Ion - ion } \\
\text { neutralization }\end{array}$} & $\mathrm{H}^{-}+\mathrm{H}_{3}^{+} \rightarrow 2 \mathrm{H}_{2}$ & & [15] \\
\hline & $\mathrm{H}^{-}+\mathrm{H}_{2}^{+} \rightarrow \mathrm{H}_{2}+\mathrm{H}(\mathrm{n}>2)$ & & [25] \\
\hline & $\mathrm{H}^{-}+\mathrm{H}^{+} \rightarrow \mathrm{H}(\mathrm{n}=3)+\mathrm{H}$ & & [15] \\
\hline Ass. detachment & $\mathrm{H}+\mathrm{H}^{-} \rightarrow \mathrm{H}_{2}+\mathrm{e}$ & & [15] \\
\hline \multirow[t]{4}{*}{ Ion conversion } & $\mathrm{H}_{2}^{+}+\mathrm{H}_{2} \rightarrow \mathrm{H}_{3}^{+}+\mathrm{H}$ & $\mathrm{X}$ & {$[15,26]$} \\
\hline & $\mathrm{H}_{2}^{+}+\mathrm{H} \rightarrow \mathrm{H}^{+}+\mathrm{H}_{2}$ & & {$[15]$} \\
\hline & $\mathrm{H}^{+}+2 \mathrm{H}_{2} \rightarrow \mathrm{H}_{3}^{+}+\mathrm{H}_{2}$ & & {$[15]$} \\
\hline & $\mathrm{H}^{+}+\mathrm{H}_{2}(v>3) \rightarrow \mathrm{H}_{2}^{+}+\mathrm{H}$ & & [15] \\
\hline
\end{tabular}

This hybrid model considers all the kinetic processes summarized in Table I: ground state elastic collisions and rotational excitations of hydrogen molecules, by electron impact; e-V, E$\mathrm{V}$ through all singlet states (eventually followed by dissociation), V-V and V-T excitations/deexcitations collisions; dissociation through triplet states $\left(\mathrm{a}^{3}, \mathrm{~b}^{3}, \mathrm{c}^{3}, \mathrm{e}^{3}\right)$; attachment and ionization for $\mathrm{H}_{2}(v)$ molecules; elastic, excitation/de-excitation and ionization collisions for $\mathrm{H}(\mathrm{n})$ atoms; wall de-excitation/recombination for both $\mathrm{H}_{2}(v>0)$ molecules (assuming a probability of 0.07 [14]) and $\mathrm{H}(\mathrm{n})$ atoms (taking a probability of $10^{-2}$ for $\mathrm{H}(1)$ [20] and of 1.0 for $\mathrm{H}(\mathrm{n}>1)$ [18]); electron-ion recombination (including wall recombination with unity probability); ion-ion neutralization (including detachment); and ion conversion reactions. Note that the cross sections for the various electron-neutral collisional processes (appearing in the electron Boltzmann equation) were normalized in order to yield a good fit between calculated and measured electron transport parameters [27,28]. Further note that we are adopting a H- 
atom wall-recombination probability, which was measured under actual discharge conditions, for an aluminum driven electrode [20].

The crm solves the set of coupled rate balance equations for the different neutral species considered here. In order to limit the calculation run times (due to the high number of species and kinetic processes involved), we have adopted zero-dimensional (0D) versions of the continuity equation for each species, by averaging them in space as follows

$$
\frac{\partial \bar{n}_{i}}{\partial t}=\left(\frac{\partial \bar{n}_{i}}{\partial t}\right)_{\text {coll-rad }}-\left(\frac{\partial \bar{n}_{i}}{\partial t}\right)_{\text {wall }}=0
$$

Here, $\left(\partial \bar{n}_{i} / \partial t\right)_{\text {coll-rad }}$ represents the average net gain rate, due to collisional-radiative mechanisms, of species $\mathrm{i}$ in the volume; $\left(\partial \bar{n}_{i} / \partial t\right)_{\text {wall }}$ represents the average net loss rate of species $i$ at the wall; and $\bar{n}_{i}$ is the average density defined as

$$
\bar{n}_{i}=\frac{\int_{0}^{d} \int_{0}^{R} n_{i} r d r d z}{\int_{0}^{d} \int_{0}^{R} r d r d z}
$$

The integrals in equation (2) distinguish between two plasma regions: a spatially homogeneous one, corresponding to the plasma bulk; a boundary layer, with size equal to the thickness of the plasma sheath, where the densities of neutral species are assumed to vary linearly. This assumption introduces a dependence of $\bar{n}_{i}$ on the corresponding diffusion coefficient and wallrecombination probability, through its flux boundary condition.

\subsection{Model solution}

The coupling between the different $\mathrm{cmv}$ calculation modules follows the usual procedure adopted in the kinetic modeling of such gas discharges [29]. The crm runs typically every $5 \mathrm{rf}$ periods, knowing the space-time average values of the charged particle densities and of the rate coefficients for the production/destruction of each neutral species. The crm non-linear equations are solved using a semi-implicit Gauss-Seidel relaxation technique. Convergence is achieved after several thousand iterations, ensuring relative variations of less than $10^{-12}$ for each neutral species density. The new chemical composition of the gas phase, obtained within the crm, is then used as input data to the homogeneous Boltzmann code, yielding an updated 
set of electron transport parameters and rate coefficients. The latter are finally used to obtain a self-consistent solution to the charged particle transport model, by adopting the local electron mean energy approximation. The charged particle transport equations are discretized in a 16x32 point grid by using second-order finite differences, and are solved for typical 1000 time steps within each $\mathrm{rf}$ period. In general, a few hundred $\mathrm{rf}$ cycles are needed to meet the convergence criterion: relative changes of particle densities, electron mean energy, plasma potential and self-bias voltage, between two consecutive periods, less than $0.05 \%$.

\section{Results and discussion}

\subsection{General features}

Figure 1 shows typical axial profiles (at $r=0$ ) of the time-average, steady-state charged particle densities, obtained at $f=13.56 \mathrm{MHz}, V_{\mathrm{rf}}=200 \mathrm{~V}$ and $p=0.5$ Torr.

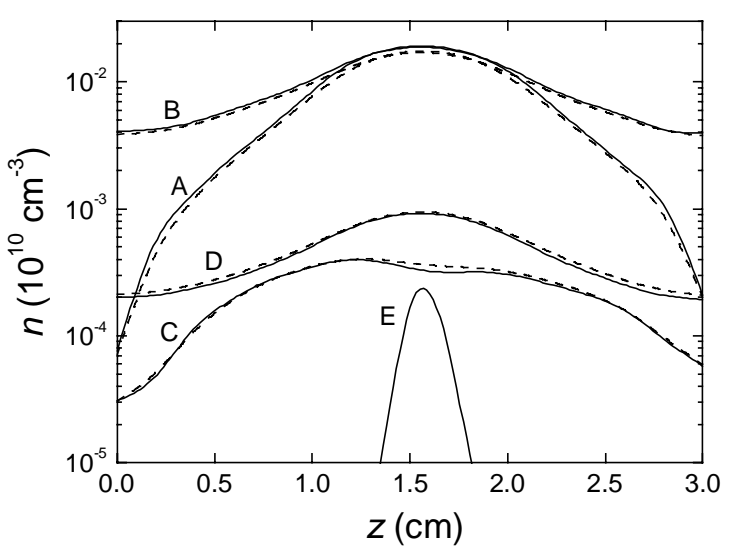

FIG.1. Axial profile (at $r=0$ ) of the time-average, steady-state particle densities, for electrons (A); positive ions $\mathrm{H}_{3}{ }^{+}(\mathrm{B}), \mathrm{H}_{2}{ }^{+}(\mathrm{C})$ and $\mathrm{H}^{+}$(D); negative ions $\mathrm{H}^{-}$(E). Results were obtained using either the cmv (solid curves) or the smv (dashed), at $f=13.56 \mathrm{MHz}, V_{\mathrm{rf}}=200 \mathrm{~V}$ and $p=0.5$ Torr.

Note that: (i) the density profiles exhibit a peak located approximately midway between the two electrodes, as a result of the alternating applied voltage; (ii) the electron density shows very steep gradients in the sheath regions (near the electrodes), due to the electric confinement produced by the rf field; (iii) the dominant ion is $\mathrm{H}_{3}{ }^{+}$, its maximum density being more than one order of magnitude higher than the mean densities of either $\mathrm{H}_{2}^{+}$or $\mathrm{H}^{+}$. This result is a direct consequence of the very efficient ion conversion reaction of $\mathrm{H}_{2}{ }^{+}$into $\mathrm{H}_{3}{ }^{+}$(see Table I); 
the $\mathrm{H}^{-}$density is smaller than the electron density, by about two orders of magnitude. Negative ions remain confined to a position located midway between the electrodes, where the plasma potential is higher, as they are mainly produced at the sheath edge, away from the discharge walls.

For comparison purposes, the results in Fig.1 were obtained using either the cmv or the smv. Although this figure shows that the use of a very complete hydrogen kinetics has little influence in the charged particle density profiles, this conclusion can not be generalized to other working conditions, as will be demonstrated later on. Note also that only by using the $\mathrm{cmv}$ it is possible to detail the main kinetic features of hydrogen in ccvhf discharges, like its vibrational distribution function (vdf) or its dissociation degree.

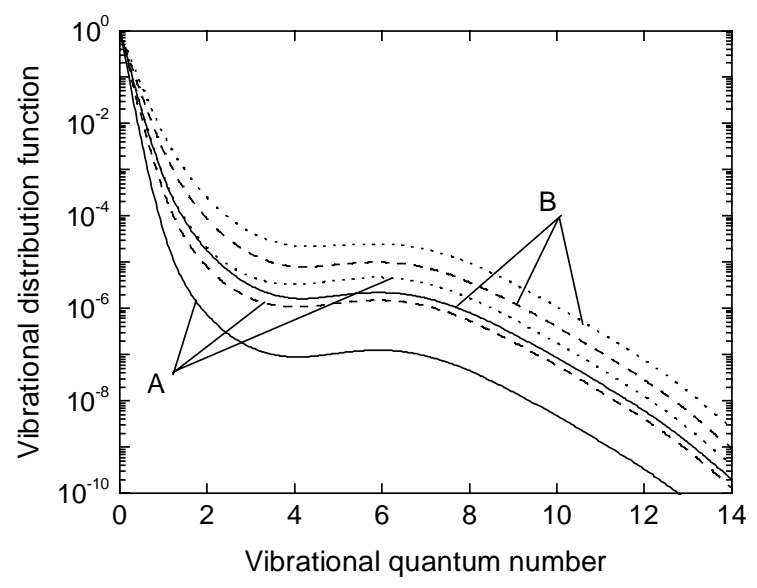

FIG. 2. Vibrational distribution function of hydrogen molecules, as calculated from the $\mathrm{cmv}$ at $p=0.3$ Torr, for $f=13.56 \mathrm{MHz}(\mathrm{A})$ and $40.68 \mathrm{MHz}(\mathrm{B})$, and the following $V_{\mathrm{rf}}$ values: $50 \mathrm{~V}$ (solid curves); $100 \mathrm{~V}$ (dashed); $200 \mathrm{~V}$ (dotted).

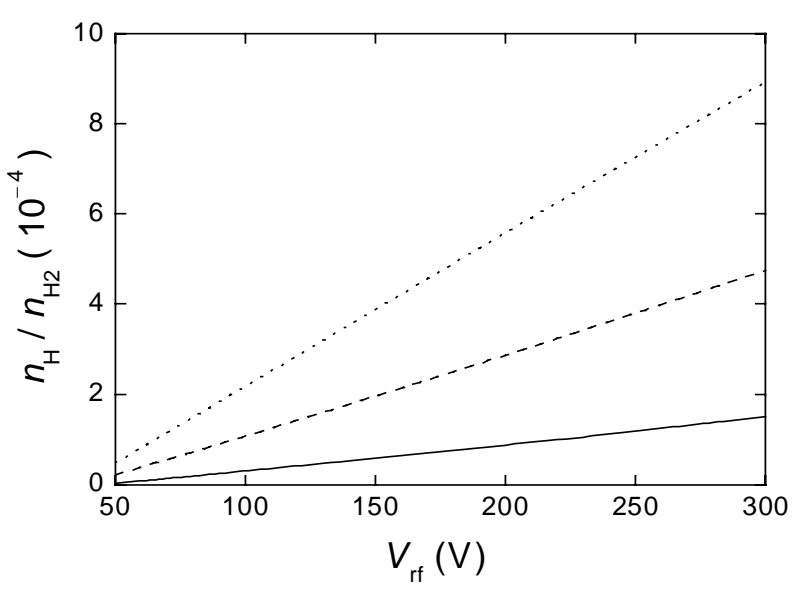

FIG. 3. Dissociation degree of hydrogen molecules, as a function of $V_{\mathrm{rf}}$, as obtained from the $\mathrm{cmv}$ at $p=0.3$ Torr and the following $f$ values: $13.56 \mathrm{MHz}$ (solid curves); $27.12 \mathrm{MHz}$ (dashed); $40.68 \mathrm{MHz}$ (dotted).

Figure 2 represents the vdf of hydrogen molecules, as calculated from the cmv for $p=0.3$ Torr, $V_{\mathrm{rf}}=50,100,200 \mathrm{~V}$ and $f=13.56,40.68 \mathrm{MHz}$, whereas Fig.3 plots the ratio of the atomic density to the molecular density of hydrogen, $n_{\mathrm{H}} / n_{\mathrm{H} 2}$, as a function of $V_{\mathrm{rf}}$, obtained from cmv simulations at $p=0.3$ Torr and $f=13.56,27.12,40.68 \mathrm{MHz}$. From these figures one observes that both the vibrational excitation and the dissociation of hydrogen are favored by an increase in either the rf applied potential or the excitation frequency. In fact, both of these changes lead to higher electric fields within discharge sheaths (hence, higher effective electrical powers 
coupled to the plasma), in order to limit the electron displacement and thus the electron wall losses [4]. Note, in Fig.2, the effect of the superelastic vibrational pumping, which results in an enhancement of the vdf's tail.

\subsection{Modeling vs. experiment}

Figure 4 shows the variation of the time-average electron density with the rf applied voltage, on the axis of the discharge and midway between the two electrodes $n_{e}(0, d / 2)$, for pressure $p=0.3$ Torr and for multiple operating frequencies $f=13.56,27.12,40.68$, and $80 \mathrm{MHz}$. The simulation results presented in this figure were obtained using either the $\mathrm{cmv}$ or the smv, to show that the updated hydrogen kinetics adopted here yields higher electron densities. The latter is mainly due to the introduction of electron production mechanisms involving $\mathrm{H}$-atoms, such as associative ionization (AI) and associative detachment (AD), which become highly competitive with respect to the ionization of hydrogen (atomic or molecular) by electron impact. Notice that the updated hydrogen kinetics features the dissociation attachment and the recombination of $\mathrm{H}_{3}{ }^{+}$ions as main electron destruction mechanisms.

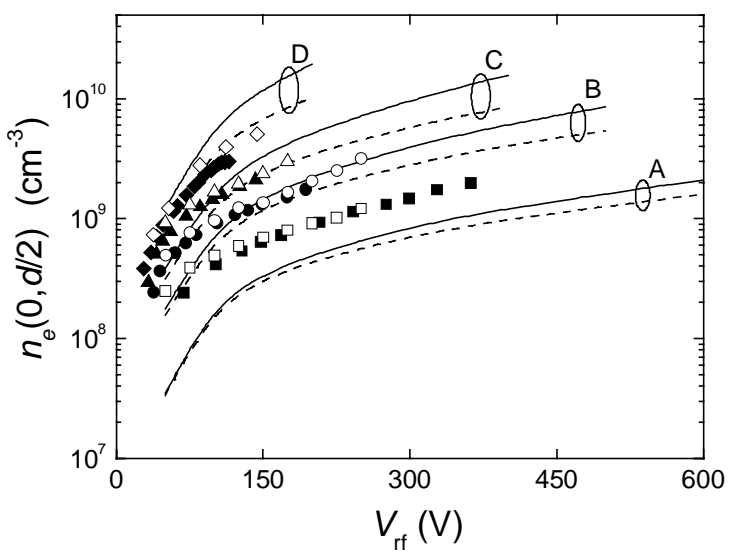

FIG.4. Time-average electron density (at $r=0$ and $z=d / 2$ ) as a function of $V_{\mathrm{rf}}$. The curves are calculation results obtained with the cmv (solid curves) and the smv (dashed), at $p=0.3$ Torr and the following frequency values: $13.56 \mathrm{MHz}$ (A); $27.12 \mathrm{MHz}$ (B); $40.68 \mathrm{MHz}$ (C); $80 \mathrm{MHz}$ (D). The points correspond to experimental measurements, obtained using a planar probe (closed points) and a cylindrical Langmuir probe (open), at frequencies $13.56 \mathrm{MHz}$ (squares), 27.12 MHz (circles), 40.68 MHz (up triangles), and $80 \mathrm{MHz}$ (diamonds). The experimental values were divided by a factor of 3 for representation purposes. 
From Fig. 4 it is observed that $n_{e}$ increases with both $V_{\mathrm{rf}}$ and $f$ (and that this increase is intensified when the cmv is used in calculations), as a consequence of the enhanced energy transfer between the rf electric field and the electron plasma population. This figure also compares simulation results to experimental measurements obtained using either a planar probe or a cylindrical Langmuir probe [5]. The planar probe (with $0.25 \mathrm{~cm}^{2}$ surface) received a $-30 \mathrm{~V}$ bias, to collect an ion flux. The corresponding electron density was obtained by using Bohm's theory (assuming a non-collisional sheath), for a constant $3 \mathrm{eV}$ electron temperature (in coherence with cylindrical probe results). Measurements of the electron density using a Langmuir probe were first obtained using the Smartprobe ${ }^{\circledR}$ tool of Scientific Systems. This tool, however, leads to incorrect results when applied to hydrogen discharges at pressures above 0.1 Torr, and thus we have directly obtained the electron temperature and density from the corresponding probe characteristics, following the techniques described in Refs. [30,31]. Although a good qualitative agreement is found between simulations and experiment, and despite the fact that the model yields higher electron densities when the complete hydrogen kinetics is adopted, the calculated values of the electron densities are still below the measured ones by a factor of 1.5-6, particularly at low applied voltages and frequencies (note that the experimental points in Fig. 4 were divided by a factor of 3). This disagreement is most probably associated to the incomplete description of the discharge space-charge sheaths, for example due to the absence of the nonlinear inertia term in the electron flux equation [3].

Figure 5 plots the effective electrical power coupled to the plasma [3], as a function of the rf applied voltage, for pressure $p=0.3$ Torr and different frequency values. It is observed that the use of the very complete hydrogen kinetics has only a little influence in the values of $W_{\text {eff. }}$ In general, the cmv leads to $W_{\text {eff }}$ values higher than the ones obtained with the smv (see also the zoom in Fig.5), probably due to the increase in the number of energy absorption channels. Note, however, that the cmv predictions for $W_{\text {eff }}$ are below the corresponding smv predictions, for frequencies above $40.68 \mathrm{MHz}$ and $\mathrm{rf}$ voltages above $150 \mathrm{~V}$, for which a more effective energy transfer takes place. As expected, $W_{\text {eff }}$ increases with both $V_{\text {rf }}$ and $f$, as a direct result of the more intense total voltages $\left[V_{\mathrm{dc}}+V_{\mathrm{rf}} \cos (2 \pi f t)\right]$ and currents $\left[I_{\mathrm{rf}}(\mathrm{t})\right]$ developed under these circumstances. This figure also shows a good agreement between model predictions and experimental measurements for $W_{\text {eff, }}$ obtained over a large range of working conditions.

The experimental results depicted in Fig.5 were obtained using a subtractive method [32], which proceeds as follows: (i) for given discharge working conditions, we measured the 
plasma-on generator power input, $P_{\mathrm{on}}$, and the rf voltage and current, $V_{\mathrm{rf}}$ and $I_{\mathrm{rf}}$; (ii) for a plasma-off situation (i.e., by operating the plasma reactor under vacuum conditions), we searched for the generator power input, $P_{\text {off }}$, which ensures the same $I_{\mathrm{rf}}$ value as measured before. Contrarily to classical methods, we used here the rf current (instead of the rf voltage), as calibration parameter; (iii) the effective electrical power coupled to the plasma was calculated as $W_{\text {eff }}=P_{\text {on }}-P_{\text {off. }}$

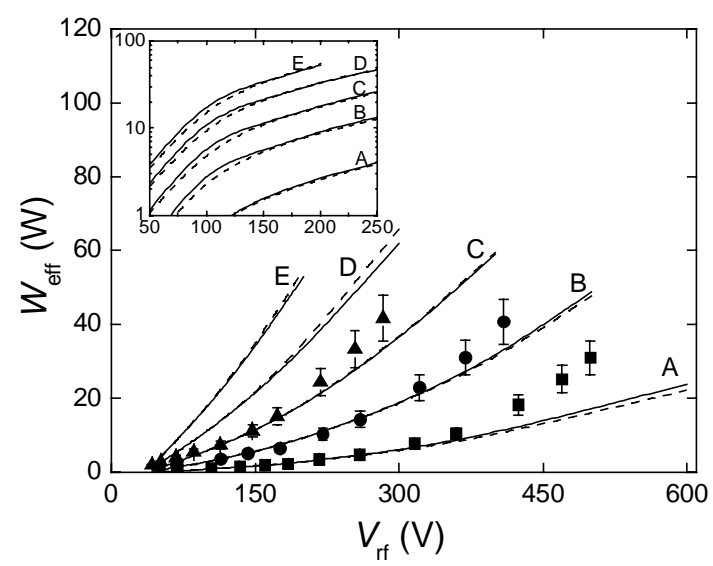

FIG.5. Effective electrical power coupled to the plasma as a function of $V_{\mathrm{rf}}$. The curves are calculation results obtained with the cmv (solid curves) and the smv (dashed), at $p=0.3$ Torr and the following frequency values: $13.56 \mathrm{MHz}$ (A); 27.12 MHz (B); 40.68 MHz (C); $60 \mathrm{MHz}$ (D); $80 \mathrm{MHz}$ (E). The points correspond to experimental measurements obtained at frequencies 13.56 MHz (squares), 27.12 MHz (circles), and 40.68 MHz (up triangles).

The values of $W_{\text {eff }}$ so obtained were validated through an alternative experimental procedure, based on an electrical model of the discharge and of its matching network, which uses as input data the measured values of $V_{\mathrm{rf}}, I_{\mathrm{rf}}$ and the phase-shift between them. Although a good agreement was found, for $f=13.56-40.68 \mathrm{MHz}$, between the values of $W_{\text {eff }}$ obtained with the subtractive and the electrical model methods, these experimental procedures yield quite different results for frequencies above $60 \mathrm{MHz}$, probably due to an incomplete discharge description within the electrical model.

Figures 6(a) and (b) represent, respectively, the time-average plasma potential $V_{p}$ at position $(r=0, z=d / 2)$ and the self-bias voltage $V_{\mathrm{dc}}$, as a function of the rf applied voltage, for pressure $p=0.3$ Torr and different frequency values. The experimental values of the plasma potential were obtained from the energy distribution function of the $\mathrm{H}_{3}{ }^{+}$ions impinging on the grounded 
electrode, as they perform very few collisions along the bulk-to-wall path. The $\mathrm{H}_{3}{ }^{+}$energy distribution function was measured using a mass spectrometer equipped with an energy analyzer, as described in [33].
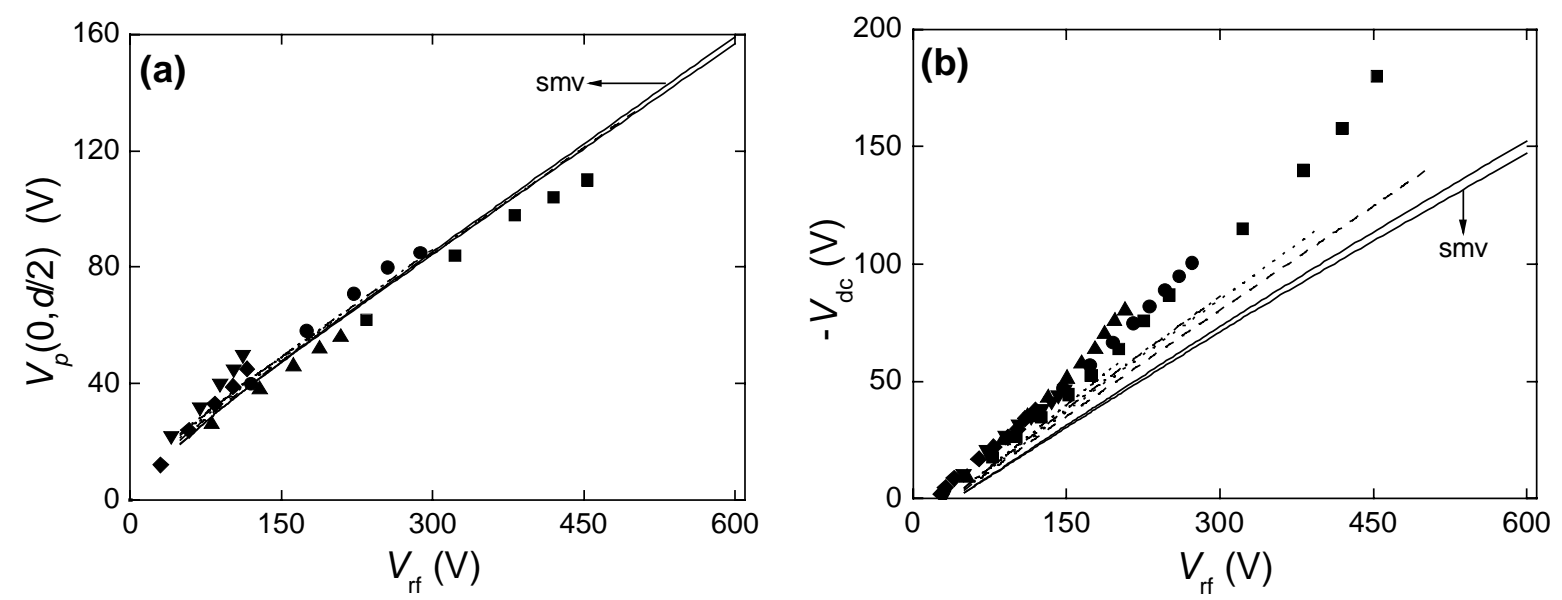

FIG.6 Electrical parameters as a function of $V_{\mathrm{rf}}$ : time-average, steady-state plasma potential at $r=0$ and $z=d / 2, V_{p}(0, d / 2)$ (a); self-bias voltage, $V_{\mathrm{dc}}(\mathbf{b})$. The curves are cmv calculation results, obtained at $p=0.3$ Torr and the following frequency values: $13.56 \mathrm{MHz}$ (solid curves); 27.12 MHz (dashed); $40.68 \mathrm{MHz}$ (dotted); $60 \mathrm{MHz}$ (dashed-dotted); $80 \mathrm{MHz}$ (dashed-dotted-dotted). The curves labeled smv were obtained at $p=0.3$ Torr and $f=13.56 \mathrm{MHz}$, using the simplified model version. The points correspond to experimental measurements obtained at frequencies 13.56 MHz (squares), 27.12 MHz (circles), 40.68 MHz (up triangles), $60 \mathrm{MHz}$ (down triangles), and $80 \mathrm{MHz}$ (diamonds).

The latter figures show that: (i) the use of the very complete hydrogen kinetics has a small influence in the values of both $V_{p}$ and $V_{\mathrm{dc}}$ (for demonstration purposes, Figs.6(a) and (b) only plot smv predictions obtained at $f=13.56 \mathrm{MHz}$ ); (ii) $V_{p}$ varies very little with $f$ being an increasing function of $V_{\mathrm{rf}}$; (iii) discharge symmetry (corresponding to smaller $V_{\mathrm{dc}}$ absolute values) is favored by a reduction in both $V_{\text {rf }}$ and $f$ values; (iv) a good agreement is found between simulation results and experimental measurements for $V_{p}$, while there is a systematic underestimation of the calculated $V_{\mathrm{dc}}$ absolute values with respect to experiment, particularly for high $V_{\text {rf }}$. The latter deviations show that the model overestimates the ion current at the rf electrode (or, alternatively, that it underestimates the corresponding electron current), thus giving a strong indication that the fluid description of the rf sheath has not yet been fully achieved. 
The coupled electrical power is plotted in Fig. 7 as a function of pressure, at fixed $V_{\mathrm{rf}}=100 \mathrm{~V}$ and for different operating frequencies. Once again, a good agreement is found between model predictions and experimental measurements for $W_{\text {eff, }}$ over a large range of discharge operating conditions (notice that, in general, this good agreement is also observed for $\mathrm{rf}$ applied voltages up to $300 \mathrm{~V}$ ). Figure 7 shows that $W_{\text {eff }}$ increases with $p$, for pressures up to 1 Torr. This increase is associated to the enhancement of the rf electric field within the discharge sheaths, induced by the compression of these charge separation regions.

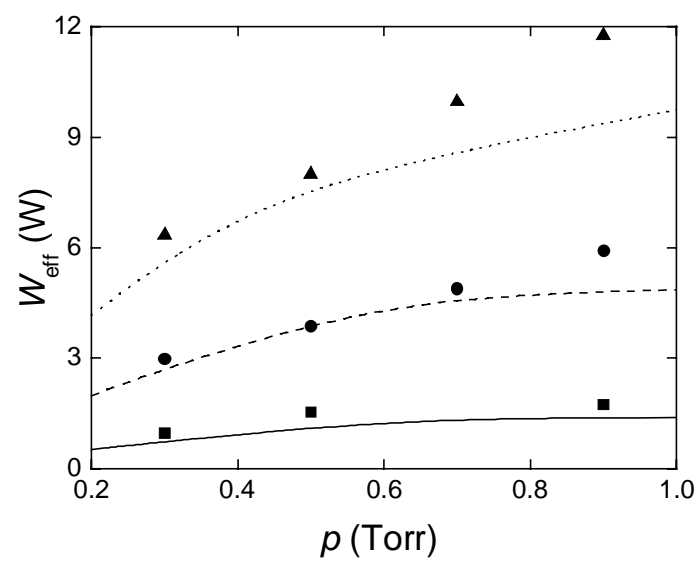

FIG.7 Effective electrical power coupled to the plasma as a function of $p$. The curves are cmv calculation results, obtained at $V_{\mathrm{rf}}=100 \mathrm{~V}$ and the following frequency values: $13.56 \mathrm{MHz}$ (solid curves); $27.12 \mathrm{MHz}$ (dashed); $40.68 \mathrm{MHz}$ (dotted). The points correspond to measurements obtained at frequencies $13.56 \mathrm{MHz}$ (squares), 27.12 MHz (circles), and 40.68 $\mathrm{MHz}$ (up triangles).

Figures $8(\mathrm{a})$ and (b) represent, respectively, the time-average plasma potential $V_{p}$ at position $(r=0, z=d / 2)$ and the self-bias voltage $V_{\mathrm{dc}}$, as a function of pressure, at $V_{\mathrm{rf}}=100 \mathrm{~V}$ and for different frequency values. From Fig.8(a) one observes that $V_{p}$ is a decreasing function of $p$, for the range of pressures analyzed here [see also Fig.7], and that the relative variations of $V_{p}$ with $f$ are always smaller than $10 \%$ [see also Fig.6(a)]. Figure 8(b) shows that: (i) $V_{\mathrm{dc}}$ is a slow decreasing function of $p$, which reveals that discharge symmetry (due to sheath compression) is favored by a pressure increase; (ii) the calculated $V_{\mathrm{dc}}$ absolute values are underestimated with respect to experiment [similarly to what was shown in Fig.6(b)], although a good qualitative agreement is observed between simulations and measurements. 

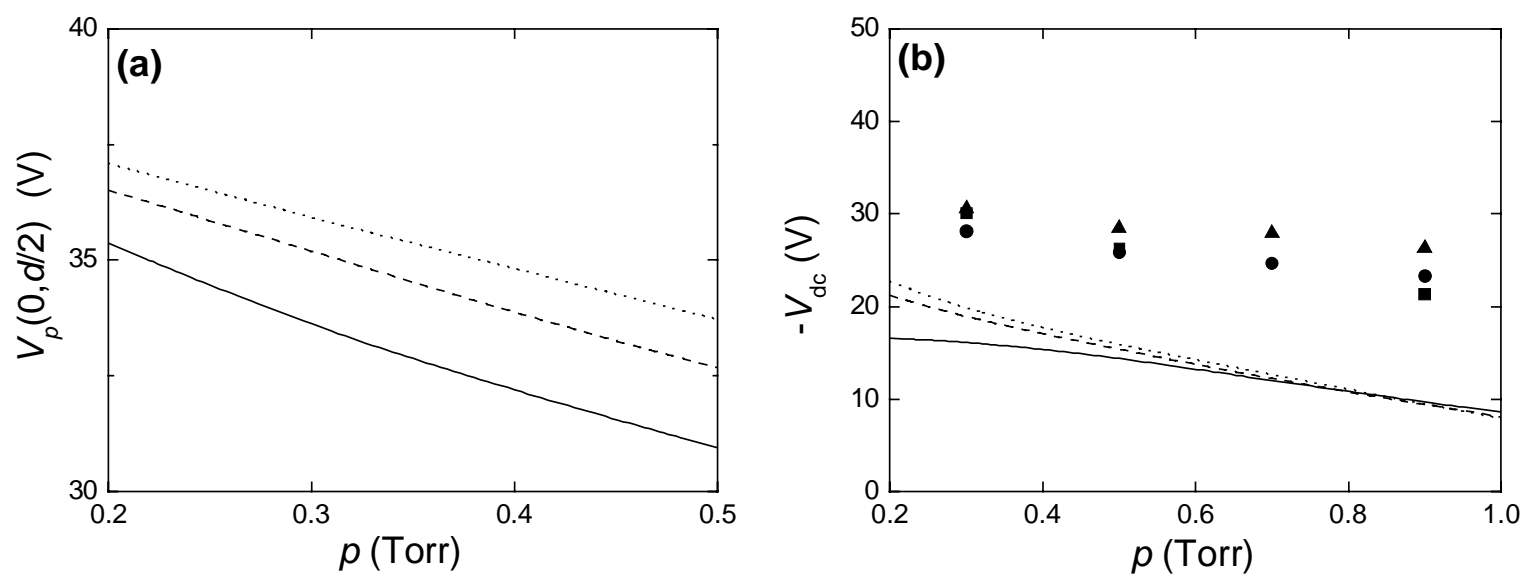

FIG.8 Electrical parameters as a function of pressure: time-average, steady-state plasma potential at $r=0$ and $z=d / 2, V_{p}(0, d / 2)$ (a); self-bias voltage, $V_{\mathrm{dc}}(\mathbf{b})$. The curves are cmv calculation results, obtained at $V_{\mathrm{rf}}=100 \mathrm{~V}$ and the following frequency values: $13.56 \mathrm{MHz}$ (solid curves); $27.12 \mathrm{MHz}$ (dashed); $40.68 \mathrm{MHz}$ (dotted). The points in Fig.8(b) correspond to measurements obtained at frequencies $13.56 \mathrm{MHz}$ (squares), $27.12 \mathrm{MHz}$ (circles), and 40.68 $\mathrm{MHz}$ (up triangles).

Figure 9 shows the variation of the time-average electron density with pressure, on the axis of the discharge and midway between the two electrodes $n_{e}(0, d / 2)$, obtained at $V_{\mathrm{rf}}=100 \mathrm{~V}$ and for $f=27.12,40.68$, and $60 \mathrm{MHz}$. The simulation results presented in this figure were obtained using either the smv or the $\mathrm{cmv}$ to confirm that the extra reactions included in the complete hydrogen kinetics do lead to higher electron densities (see also Fig.4). However, as before, the calculated values of the electron density are still below the measured ones by a factor of 1.5-5 (in Fig.9, the experimental points were once again divided by a factor of 3). Figure 9 also shows that the electron density varies very little within the range of pressures analyzed here, when using the smv (no H-atoms considered), whereas a strong increase of $n_{e}$ with $p$ is observed, when using the cmv. The latter is due to the introduction of electron production mechanisms involving $\mathrm{H}$-atoms, such as $\mathrm{AI}$ and $\mathrm{AD}$, whose relative importance is enhanced with pressure increase. In fact, the electron production rates via $\mathrm{AI}$ or $\mathrm{AD}$, at position $(0, d / 2)$, present a limited variation (of less than a factor of 2 for AI and of less than a factor of 30 for $\mathrm{AD}$ ), over the pressure range considered here, whereas the ionization rate of hydrogen (atomic or molecular) by electron impact becomes highly attenuated at $(0, d / 2)$, being strongly enhanced within discharges sheaths, due to the decrease in $V_{p}$ as $p$ increases. 


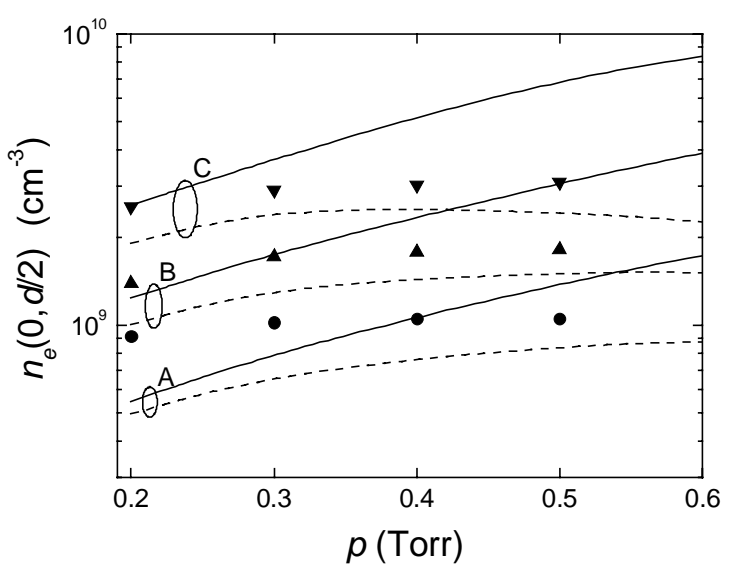

FIG.9. Time-average electron density (at $r=0$ and $z=d / 2$ ) as a function of $p$. The curves are calculation results obtained with the cmv (solid curves) and the smv (dashed), at $V_{\mathrm{rf}}=100 \mathrm{~V}$ and the following frequency values: $27.12 \mathrm{MHz}(\mathrm{A}) ; 40.68 \mathrm{MHz}$ (B); $60 \mathrm{MHz}$ (C). The points correspond to experimental measurements, obtained using a cylindrical Langmuir probe at frequencies $27.12 \mathrm{MHz}$ (circles), $40.68 \mathrm{MHz}$ (up triangles), and $60 \mathrm{MHz}$ (down triangles). The experimental values were divided by a factor of 3 for representation purposes.

Figure 9 evidences a disagreement between experimental measurements (which show negligible variations of $n_{e}$ with $p$ ) and cmv predictions, which might indicate that the model is not providing a good estimate for the H-atoms density, particularly at higher pressures. To further investigate this problem, we plot in Fig.10 the time-average $\mathrm{H}$-atoms density $n_{\mathrm{H}}$, as a function of pressure, obtained at constant $W_{\mathrm{eff}}=30 \mathrm{~W}$ and for $f=13.56,27.12$, and $40.68 \mathrm{MHz}$. For comparison purposes, the simulation results presented in this figure were obtained by running the $\mathrm{cmv}$ at two different values of the wall-recombination probability: $\gamma_{\mathrm{H}}=10^{-2}$ and 0.2 . The H-atom density has been measured by using two-photon absorption laser-induced fluorescence (TALIF) diagnostics [20]. The second harmonic of a Nd-Yag laser at $532 \mathrm{~nm}$ was used to pump a dye mixture of R610 and DCM, in order to produce a laser emission at $615 \mathrm{~nm}$. The third harmonic of the $615 \mathrm{~nm}$ fundamental wavelength (obtained from frequency doubling in a KDP crystal followed by frequency mixing in a BBO crystal) was used to produce a 205 $\mathrm{nm}$ laser emission. This laser beam was focused onto the plasma chamber with a lens of $57 \mathrm{~cm}$ focal length. The resulting induced fluorescent radiation was collected through the reactor grid by a lens, was imaged on the entrance slit of a monochromator, and it was detected by a photomultiplier connected to a BOXCAR averager. The absolute $\mathrm{H}$-atom density was derived 
from the measurements of the generated TALIF signal, on the axis of the discharge at $12 \mathrm{~mm}$ from the driven electrode, using a well-known $\mathrm{Kr}: \mathrm{H}$ detection sensitivity ratio [34].

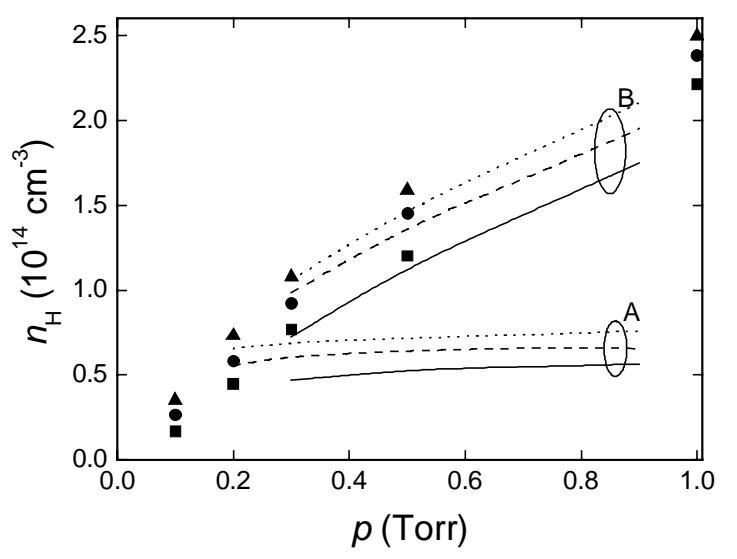

FIG.10. Time-average $\mathrm{H}$-atoms density as a function of $p$. The curves are cmv calculation results, obtained at $\gamma_{\mathrm{H}}=10^{-2}(\mathrm{~A})$ and $\gamma_{\mathrm{H}}=0.2(\mathrm{~B})$, for $W_{\mathrm{eff}}=30 \mathrm{~W}$ and the following frequency values: $13.56 \mathrm{MHz}$ (solid curves); 27.12 MHz (dashed); $40.68 \mathrm{MHz}$ (dotted). The points were obtained by TALIF diagnostics, at frequencies $13.56 \mathrm{MHz}$ (squares), 27.12 MHz (circles), and 40.68 $\mathrm{MHz}$ (up triangles). Curves B were multiplied by a factor of 10 for representation purposes.

As anticipated, Fig.10 presents a significant disagreement between measurements (showing a strong increase of $n_{\mathrm{H}}$ with $p$ ) and cmv calculations at $\gamma_{\mathrm{H}}=10^{-2}$ (showing negligible variations of $n_{\mathrm{H}}$ with $p$ ), although both experiment and simulations yield the same order of magnitude for the $n_{\mathrm{H}}$ density. As expected, the $\mathrm{H}$-atoms density is strongly reduced when calculations adopt a higher wall-recombination probability of 0.2 (note that, in this case, simulation results were multiplied by a factor of 10).

The calculated $n_{\mathrm{H}}$ vs. $p$ variation, obtained with the cmv at $\gamma_{\mathrm{H}}=10^{-2}$, and its disagreement with respect to experiment can be explained by a combination of three factors. First, the cmv includes a 0D crm that imposes a flat profile for the bulk density of all neutral species (and in particular of the H-atoms, see Sec. 2.2), regardless of the working pressure. Most probably, this assumption is not valid at high pressures, in which case the neutral species normally exhibit a non-homogeneous density profile, associated to diffusion transport. Furthermore, the differences between bulk and wall densities become highly attenuated at low- $\gamma_{\mathrm{H}}$, as the flux boundary condition relating the boundary layer to the plasma bulk goes to zero (in our case, 
this yields a constant density profile all across the discharge, from the bulk to the wall). The latter considerations might explain the good qualitative agreement between measurements and simulations at $\gamma_{\mathrm{H}}=0.2$. Second, model predictions for the energy density $n_{e} \varepsilon$ (where $\varepsilon$ represents the electron mean energy) remain practically unchanged with modifications on the reaction kinetics. Consequently, an increase in $n_{e}$ (for example, following the introduction of a very complete hydrogen kinetics) leads to a simultaneous decrease in $\varepsilon$, in order to keep the same $n_{e} \varepsilon$ values. In particular, smv calculations yield $\varepsilon=5-2.5 \mathrm{eV}$ (at the center of the discharge, and for pressures between 0.3 and 0.9 Torr), whereas $\mathrm{cmv}$ predictions (with a complete updated kinetic scheme) give $\varepsilon=5-1 \mathrm{eV}$, for the same pressure region. This reduction in the electron mean energy: (i) might be caused by missing electron energy gain terms in the corresponding energy balance equation; (ii) will contribute to a strong decrease in the H-atoms production rate (particularly at high pressures), which mainly proceeds via the dissociation of $\mathrm{H}_{2}$ by electron impact. Third, the experimental results for the H-atoms density, presented in Fig.10, were obtained at constant generator power input, $P_{\mathrm{on}}=30 \mathrm{~W}$ (not constant coupled electrical power, $W_{\text {eff }}=30 \mathrm{~W}$ ). In general, the energy coupling is more effective at high pressures, and so one can expect an underestimation of low-pressure $n_{\mathrm{H}}$ measurements, with respect to calculations obtained at constant $W_{\text {eff. }}$

\section{Final remarks}

This paper has compared numerical simulations to experimental measurements of various plasma parameters, in view of the characterization of capacitively coupled hydrogen discharges, produced within a parallel plate cylindrical setup at different frequencies $(f=13.56-$ $80 \mathrm{MHz})$, pressures $(p=0.2-1$ Torr $)$, and $\mathrm{rf}$ applied voltages $\left(V_{\mathrm{rf}}=50-600 \mathrm{~V}\right)$. A charged particle transport model was self-consistently coupled with a homogeneous kinetic model for hydrogen, including vibrationally excited molecular species and electronically excited atomic species. This updated hydrogen kinetics led to higher electron densities, due to the presence of electron production mechanisms involving $\mathrm{H}$-atoms, such as associative ionization and associative detachment. A good quantitative agreement was found between calculation and experiment for the coupled electrical power and the plasma potential, at various frequencies, pressures and applied voltages. However, the model has generally underestimated the electron density and the self-bias potential with respect to measured values, which probably indicates that the fluid description of rf space-charge sheaths is still incomplete. Model predictions for 
the absolute density of $\mathrm{H}(\mathrm{n}=1)$ atoms (which show negligible variations of $n_{\mathrm{H}}$ with $p$, at various frequencies) were compared to TALIF diagnostic results (which show a strong increase of $n_{\mathrm{H}}$ with $p$, for the same frequencies). This disagreement between simulations and measurements is probably associated to the use of a $0 \mathrm{D} \mathrm{crm}$, together with an incomplete electron energy description when using the updated hydrogen kinetics. Work is in progress to overcome these model limitations.

\section{Acknowledgements}

L. Marques acknowledge the financial support of the Portuguese Foundation for Science and Technology (FCT), under fellowship SFRH/BD/5012/2001.

\section{References}

[1] M. Hertl, J. Jolly and G. Baravian, "Detection of hydrogen atoms in $\mathrm{SiH}_{4}-\mathrm{H}_{2}$ radiofrequency plasmas using two-photon laser-induced fluorescence", J. Appl. Phys. 92, 710-715 (2002)

[2] O. Leroy, G. Gousset, L.L. Alves, J. Perrin and J. Jolly, "Two-dimensional modeling of $\mathrm{SiH}_{4}-\mathrm{H}_{2}$ radiofrequency discharges for a-Si:H deposition", Plasma Sources Sci. Technol. 7, 348-358 (1998)

[3] A. Salabas, G. Gousset and L.L. Alves, "Two-dimensional fluid modeling of charged particle transport in radio-frequency capacitively coupled discharges”, Plasma Sources Sci. Technol. 11, 448-465 (2002)

[4] A. Salabas, L. Marques, J. Jolly, G. Gousset and L.L. Alves, "Systematic characterization of low-pressure capacitively coupled hydrogen discharges", J. Appl. Phys. 95, 4605-4620 (2004)

[5] L. Marques, A. Salabas, G. Gousset, J. Jolly and L.L. Alves, "Modelling the influence of frequency in a low pressure capacitively coupled hydrogen discharge", in Proceedings of the $16^{\text {th }}$ ESCAMPIG and $5^{\text {th }}$ ICRP, edited by N. Sadeghi and H. Sugai (Grenoble, France, 2002) pp. $69-70$

[6] L. Marques, A. Salabas, G. Gousset, J. Jolly and L.L. Alves, "Influence of vibrational kinetics in a low pressure radio-frequency hydrogen discharge", in CD-proceedings of the $8^{\text {th }}$ ISPC, edited by R. D’Agostino, P. Favia, F. Fracassi and F. Palumbo (Taormina, Italy, 2003) 
[7] L. Marques, J. Jolly, G. Gousset and L.L. Alves, "Modelling of capacitively coupled hydrogen discharges: the role of hydrogen kinetics", submitted to the $17^{\text {th }}$ ESCAMPIG, (Constanta, Romania, 2004)

[8] H. Tawara, Y. Itikawa, H. Nishimura and M. Yoshino, "Cross sections and related data for electron collisions with hydrogen molecules and molecular ions”, J. Phys. Chem. Ref. Data 19, 617-636 (1990)

[9] J. Loureiro and C.M. Ferreira, "Electron and vibrational kinetics in the hydrogen positive column”, J. Phys. D 22, 1680-1691 (1989)

[10] C. Gorse, M. Capitelli, M. Bacal, J. Bretagne and A. Laganà, "Progress in the nonequilibrium vibrational kinetics of hydrogen in magnetic multicusp $\mathrm{H}^{-}$ion sources", Chem. Phys. 117, 177-195 (1987)

[11] D. Rapp and P. Englander-Golden, "Total cross sections for ionization and attachment in gases by electron impact. I. Positive ionization”, J. Chem. Phys. 43, 1464-1479 (1965)

[12] T. Simko, V. Martisovits, J. Bretagne and G. Gousset, "Computer simulations of $\mathrm{H}^{+}$and $\mathrm{H}_{3}{ }^{+}$transport parameters in hydrogen drift tubes”, Phys. Rev. E 56, 5908-5919 (1997)

[13] A.P. Hickman, "Dissociative attachment of electrons to vibrationally excited $\mathrm{H}_{2}$ ", Phys. Rev. A 43, 3495-3502 (1991)

[14] C. Gorse, M. Capitelli, J. Bretagne and M. Bacal, "Vibrational excitation and negative-ion production in magnetic multicusp hydrogen discharges”, Chem. Phys. 93, 1-12 (1985)

[15] A.A. Matveyev and V.P. Silakov, "Kinetic processes in a highly-ionized non-equilibrium hydrogen plasma”, Plasma Sources Sci. Technol. 4, 606-617 (1995)

[16] M. Mukherjee, M. Basu and A.S. Gosh, "Elastic electron-hydrogen scattering at intermediate energies", Phys. Rev. A 36, 937-940 (1987)

[17] I. Bray, D.A. Konovalov and I.E. McCarthy, "Coupled channel optical calculation of electron hydrogen scattering: elastic scattering from 0.5 to 30 eV", Phys. Rev. A 43, 58745877 (1991)

[18] G. Gousset, private communication

[19] M. Glass-Maujean, "Collisional quenching of $\mathrm{H}(2 \mathrm{~S})$ atoms by molecular hydrogen: two competitive reactions", Phys. Rev. Lett. 62, 144-146 (1989)

[20] J. Jolly and S. Dine, "Laser-induced fluorescence measurements of atomic hydrogen densities in capacitively coupled VHF plasmas in $\mathrm{H}_{2}$ ", submitted to the $17^{\text {th }}$ ESCAMPIG, (Constanta, Romania, 2004) 
[21] R.K. Janev, W.D. Langer, K. Evans and D.E. Post, "Elementary processes in hydrogen helium plasmas: cross sections and reaction rate coefficients", edited by G. Ecker, P. Lambropolous and H Wlather (Springer, Berlin, 1987)

[22] M.J. Kushner, "A model for the discharge kinetics and plasma chemistry during plasma enhanced chemical vapor deposition of amorphous silicon”, J. Appl. Phys. 63, 2532-2551 (1988)

[23] J. Perrin, O. Leroy and M.C. Bordage, "Cross-sections, rate constants and transport coefficients in silane plasma chemistry", Contrib. Plasma Phys. 36, 3-49 (1996)

[24] C. F. Chan, "Reaction cross-sections and rate coefficients related to the production of positive ions”, Lawrence Berkeley Lab. Report No. LBID-632, 1983

[25] M.J.J. Eerden, M.C.M. van de Sanden, D.K. Otorbaev and D.C. Schram, "Cross section for the mutual neutralization reaction $\mathrm{H}_{2}{ }^{+}+\mathrm{H}^{-}$, calculated in a multiple-crossing Landau-Zener approximation”, Phys. Rev. A 51, 3362-3365 (1995)

[26] L.P. Theard and W.T. Huntress, Jr., "Ion-molecule reactions and vibrational deactivation of $\mathrm{H}_{2}{ }^{+}$ions in mixtures of hydrogen and helium”, J. Chem. Phys. 60, 2840-2848 (1974)

[27] J. Dutton, “A survey of electron swarm data”, J. Phys. Chem. Ref. Data 4, 577-856 (1975)

[28] A.V. Phelps, ftp://jila.colorado.edu/collision_data/

[29] S. Longo and I. Boyd, "Nonequilibrium dissociation of hydrogen in a parallel-plate radio frequency discharge”, Chem. Phys. 238, 445-453 (1998)

[30] Z. Zakrzewski and T. Kopiczynski, "Effect of collisions on positive ion collection by a cylindrical Langmuir probe", Plasma Physics 16, 1195-1198 (1974)

[31] M. Tichy, M. Sicha, P. David and T. David, "A collisional model of the positive ion collection by a cylindrical Langmuir probe”, Contrib. Plasma Phys. 34, 59-68 (1994)

[32] V.A.Godyak and R.B. Piejak, "In situ simultaneous radio frequency discharge power measurements", J. Vac. Sci. Technol. A8, 3833-3837 (1990)

[33] K. Köhler, J.W. Coburn, D.E. Horne, E. Kay and J.H. Keller, "Plasma potentials of 13.56 MHz rf argon glow discharges in a planar system” J. Appl. Phys. 57, 59-66 (1985)

[34] M.G.H. Boogaarts, S. Mazouffre, G.J. Brinkman, H.W.P. van der Heijden, P. Vankan, J.A.M. van der Mullen, D.C. Schram, H.F. Döbele, "Quantitative two-photon laser-induced fluorescence measurements of atomic hydrogen densities, temperatures, and velocities in an expanding thermal plasma”, Rev. Sci. Instrum. 73, 73-86 (2002) 